\title{
An unhealthy holiday on Lake Bolsena
}

\author{
Lucio Brugioni, Maurizio Tognetti, Cristina Gozzi \\ Medicina Interna ed Area Critica, AOU Policlinico di Modena, Italy
}

\begin{abstract}
A 70-year old man (OL) was hospitalized due to fever (up to $40^{\circ} \mathrm{C}$ ) associated with malaise and abdominal pain. Tests showed hypereosinophilic syndrome, and increased liver and inflammation indexes. Abdominal echography showed a nonhomogeneous liver and the spleen was enlarged. Abdominal computed tomography showed multiple abscesses on the liver. The patient reported that he had recently gone on holiday to Lake Bolsena where he had eaten raw fish. A specialist in infectious diseases confirmed that in that area there is an infestation of Opisthorchis felineus. Analysis of blood and stool samples confirmed the presence of this parasite. The patient was treated with praziquantel with resolution of fever and improvement in clinical tests and general condition. Opisthorchis felineus is a parasite that commonly infects dogs and cats. Infection is sometimes transmitted to man and is usually related to eating raw fish. The parasite enters the liver and its eggs are found in the patient's stool samples. Acute symptoms are: fever, diarrhea, abdominal pain, and malaise. Hypereosinophilic syndrome and increase in liver index are typical. Chronic infection could result in cholangiocarcinoma. Presence of the parasite is confirmed through specific analysis of blood and stool. Therapy consists of praziquantel or, as second choice, abendazol. In the $20^{\text {th }}$ century, there were 4 epidemics in Italy, on Lakes Trasimeno and Bolsena in Central Italy. The latest epidemic was reported in that area in summer 2012.
\end{abstract}

\section{Case Report}

A 70-year old man (OL) was hospitalized due to three days of fever up to $40^{\circ} \mathrm{C}$ associated with chronic asthenia, lack of appetite, diarrhea and abdominal pain, mainly in the right hypochondrium. The patient was under therapy with $5 \mathrm{mg}$ ramipril for high blood pressure.

On admittance to hospital, the patient was alert, collaborative, distressed and in pain, with vital parameters within normal values; body temperature was $37.5^{\circ} \mathrm{C}$. Cardiorespiratory responses were normal. The abdomen was palpable but painful in the right

Correspondence: Lucio Brugioni, Medicina Interna ed Area Critica, AOU Policlinico di Modena, Italy.

Tel. +39.059.4225491.

E-mail: brugioni.lucio@policlinico.mo.it

Key words: Opisthorchis felineus, fever, hypereosinophilia, liver abscess.

This work is licensed under a Creative Commons Attribution NonCommercial 3.0 License (CC BY-NC 3.0).

CCopyright L. Brugioni et al., 2013

Licensee PAGEPress, Italy

Italian Journal of Medicine 2013; 7:39-42

doi:10.4081/itjm.2013.39 hypochondrium. Murphy's sign was negative and peristalsis was functional.

Biohumoral tests showed eosinophilic leukocytosis (WBC $16 \times 10^{9} / \mathrm{L}$, eosinophils $30 \%$ ), increase in cytolytic and hepatic cholestatic indexes (GOT/GPT 209/159 $\mu / \mathrm{L}$, alkaline phosphatase 350 $\mu / \mathrm{L}$, gamma GT $527 \mu / \mathrm{L}$ ), increase in aspecific phlogosis (ferritin $650 \mathrm{ng} / \mathrm{mL}$, PCR $15 \mathrm{mg} / \mathrm{dL}$, fibrinogen $659 \mathrm{mg} / \mathrm{dL}$ ).

Abdominal ecography showed a non-homogeneous liver and splenomegaly $(15 \mathrm{~cm})$. Abdominal computed tomography (CT) scan showed the liver to have numerous non-homogeneous hypodense areas with irregular contours compatible with hepatic abscesses. There was a small effusion into the pelvis.

Wide-spectrum antibiotic therapy was started with PIP-TAZ and a series of microvirological tests were carried out: markers for hepatitis, HIV screening (after obtaining the patient's informed consent), serological Wright and Widal tests, $\mathrm{A} / \mathrm{C}$ antibody tests for Entamoeba histolitica, Echinococcus, Leptospira, and Bartonella. Blood cultures were also taken at peaks of high fever. During his hospital stay, the patient developed diarrhea with loss of greenishcolored feces. Coproculture for Clostridium difficile, Salmonella, Shigella and parasites was negative. A further investigation into the patient's case history confirmed that 20 days earlier, during a holiday on Lake Bolsena, Central Italy, he had eaten raw fish marinated in fresh water.

A specialist in infectious diseases informed us of 
an epidemic in this area of a parasite called Opistorchis felineus. Analysis of our patient's blood and stool samples confirmed the presence of this parasite.

Treatment was, therefore, started with praziquantel. The fever disappeared, and biohumoral values and his general clinical condition improved. The patient was sent home after eight days and sent for follow up at the Infectious Diseases Outpatients Clinic.

At a 30-day check-up, the patient was in good physical condition, laboratory tests showed cytolytic values in the norm. However, cholestatic indexes had still not returned to normal.

Check-up abdominal CT still showed the presence of hepatic abscesses but there was a significant reduction in both number and size. Further parasitological tests of feces were negative.

The patient's wife had been on holiday with her husband and had also eaten the raw fish. Although asymptomatic, she was also given anti-parasite treatment. Her blood and stool samples were also positive for $O$. felineus.

The patient authorized the use of his personal data.

\section{Discussion}

Opistorchiasis is a disease caused by trematodes of the genus Opistorchis. There are essentially 3 known species: $O$. felineus, $O$. viverrini and $O$. guayaquilensis. O. viverrini are widespread in Laos and in Thailand, the felineus in Germany, Poland, the Baltic, Russia and Siberia, and the guayaquilensis in Ecuador. ${ }^{1,2}$ The trematodes are to be found in the biliary tract of dogs, cats and rats. These definitive hosts become infested after eating fish such as carp and tench that are either raw or undercooked and that are already infested with the parasite. Man is an occasional definitive host. The parasite needs 3 hosts to complete its lifecycle: 2 intermediate hosts (a fresh water snail of the genus Bithynia and a soft water fish) and one definitive host (dog, cat or man).

The eggs of the worm enter fresh water through the feces of mammals and are eaten by the snails. The eggs develop in cercariae, the parasitic larvae of a trematode worm, that then penetrate the skin of fish. From here, the cercariae enter the muscles and transform themselves into metacercariae. They later enter the definitive host in the form of food. In man, the metacercariae settle in the biliary ducts and develop into adult parasites that then lay their eggs. The eggs are carried by bile into the feces. The eggs of the $O$. sinensis are $35 \times 20 \mu \mathrm{m}$ in size, are small, fat and oval in shape, a yellowish-brown color and have a small knob or hooklike protrusion on its back. Eggs of $O$. felineus are smaller and longer in shape. Adult $O$. sinensis are 1-2 $\mathrm{cm}$ long, lance-shaped and orange in color. ${ }^{3}$
There is no risk of contagion from an infested human to other men or women. The first case of opisthorchiasis in man was described by Konstantin Wingradoff as long ago as 1892. The presentation and severity of symptoms depends on the quantity of parasites present in the organism. O. viverrini is usually less symptomatic than $O$. felineus. The most common symptoms are: fever, diarrhea, abdominal pain and also obstructive jaundice is sometimes presented. Infected individuals present hepatomegaly. Symptoms usually appear 10-25 days after eating the infected raw fish. All infected individuals present hypereosinophilia and an increase in hepatic cytolytic and cholestatic indexes. Infection sometimes becomes chronic even though symptoms can be slight and non-specific. In advanced phase, signs and symptoms related to obstruction of the biliary tract prevail. Pyogenic cholangitis, hepatic abscesses, and pancreatisis are common and portal hypertension can develop. Chronic forms also present cholangiocarcinoma. In fact, the parasites provoke chronic phlogosis of the biliary epithelium with hyperplasia, displasia and fibrosis. In countries such as Thailand where $O$. viverrini is endemic, there is a 15 -fold increased risk of cholangiocarcinoma in infected individuals. ${ }^{4,5}$

Diagnosis is not simple and cannot be intuitive but must be based on a careful evaluation of patient case history. Specific coproparassitological tests can confirm the presence of the eggs in the feces. Enzyme-linked immunosorbent assay (ELISA) can also provide further evidence of Ag in the blood, and this is also useful for the management of follow-up therapy.

Treatment is based on praziquantel $(50-75 \mathrm{mg} / \mathrm{kg}$ in 3 doses/day). However, this drug is not currently available in Italy but only in the Republic of San Marino and in the Vatican City. Second-choice therapy is albendazole $(10 \mathrm{mg} / \mathrm{kg}$ twice a day for seven days). The few side-effects experienced with this drug means that this can also be used as additional therapy in suspect cases. ${ }^{6}$

The best precautionary approach is health education aimed at avoiding consumption of raw fresh water fish unless cooked and frozen at $-20^{\circ} \mathrm{C}$ for a week; both treatments are capable of inactivating the parasite. $^{7}$

In Italy, 4 epidemics were reported during the $20^{\text {th }}$ century. All cases involved eating fish, mostly tench, from lakes in Central Italy (Lakes Trasimeno and Bolsena). The last epidemic was reported in summer 2012 and our patient was just one of the many holidaymakers infected by the parasite.

Our patient presented the following characteristics: fever, hypereosinophilia (shown by biohumoral marker) and hepatic abscesses (shown by radiological tests). 
Considering that:

- hypereosinophilia (eosinophils $>500$ cells $/ \mu \mathrm{L}$ ) associated with hyperpyrexia can be found in the course of i) infectious diseases: parasitic infections (Strongyloidiasi, Trichinosis, Filariasis), fungal infections (Aspergillosis), HIV; ii) neoplastic pathologies: leukemia, lymphoma, hypereosinophilic syndrome, cancer of the ovaries; iii) rheumatological diseases: vasculitis (Churg Strauss), systemic lupus erythematosus, Behcet's disease; iv) eosinophilic pulmonitis: groups of diseases of known or unknown causes characterized by eosinophilic pleurisy and general peripheral eosinophilia; ${ }^{8-10}$

- liver abscesses associated with hypereosinophilia can be due to a variety of causes, such as allergic diseases, parasitic infections, neoplasms and hypereosinophilic syndrome; ${ }^{11-13}$

there is still a very wide range of possible causes of a concomitant presention of fever, hypereosinophilia and liver abscesses. This case was only resolved by a quick association being made between the symptoms presented and the patient's recent stay in an area known to be at risk of parasitic epidemic.

\section{Conclusions}

Gastroenteritis usually has an infectious etiology. The most frequent cause is certainly viral (rotavirus, adenovirus, norovirus). Less common, but more serious, are bacterial forms in the course of food poisoning (from Salmonella, Shigella, Colibacilli). Parasitic forms are even rarer but still dangerous (Ameba, Giardia). It is also to be remembered that enteritis can also be a result of pharmaceutical therapy, typically from antibiotics due to the changes in the intestinal microbial flora. Forms due to different types of allergies, including food allergies, are less frequent.

In the summer, gastroenteritis is usually related to viral infections or to food poisoning. They usually follow a benign course and, except when newborns, young children, the eldery or immunodepressed subjects are involved, hospitalization is not necessary. Patients will be treated at home by their family doctor.

However, certain conditions require access to emergency units, such as: persistent diarrhea for more than 3-4 days with constant high fever that does not respond (or only slightly) to normal homebased therapy, inability to maintain adequate hydration, blood in stool samples, change in sensory response. Simple laboratory tests allow the physi- cian to evaluate whether the patient is developing acute renal sufficiency and if the loss of salt is resulting in hypokaliemia, if there are critical increases in phologistic indexes, and if the patient has become anemic. A typical case is that of elderly patients presenting viral gastroenteritis. These will continue therapy at home with diuretics, ACE inhibitors and, therefore, develop chronic renal insufficiency with severe hypokaliemia and consequent metabolic acidosis.

Significant eosinophilia associated to a striking presentation of symptoms in adult immunocompetent subjects should lead the physician to take into consideration those less frequent causes in the differential diagnostic procedure. If not treated in time, these types of infection can become life threatening. In our patient, in fact, the agent responsible was the parasite O. felineus.

In conclusion, the possibility of an infection from O. felineus should always be taken into consideration and excluded in patients who have recently spent time on Lake Bolsena or other lakes in Central Italy, and who go to their doctor with gastroenteritis. Early diagnosis and targeted therapy are essential for a rapid solution of their clinical condition.

\section{References}

1. Conlan JV, Sripa B, Attwood S. A review of parasitic zoonoses in a changing Southeast Asia. Vet Parasitol 2011;182:22-40.

2. Forrer A, Sayasone S. Spatial distribution of, and risk factor for, Opisthorchis viverrini Infection in Southern LAO. PLos Negl Trop Dis 2012;6:e1481.

3. Sohn WM, Shin EH, Yong TS. Adult Opisthorchis viverrini flukes in humans, Takeo, Cambodia. Emerg Infect Dis 2011;17:1302-4.

4. Samaras V, Rafailidis PI, Peppas G. Chronic bacterial and parasitic infections and cancer: a review. J Infect Dev 2010;267-81.

5. De Martel C, Plummer M, Franceschi S. Cholangiocarcinoma: descriptive epidemiology and risk factors. Gastroenterol Clin Biol 2010;34:173-80.

6. Schuster RK. Opisthorchiidosis: a review. Infect Dos Drug Targets 2010;10:402-15.

7. Keiser J, Duthaler U. Update on the diagnosis and treatment of food borne trematode infections. Curr Opin Infect Dis 2010;23:513-20.

8. Roufosse F, Weller PF. Practical approach to the patient with hypereosinophilia. J Allergy Clin Immunol 2010; 126:139.

9. Simon $\mathrm{Hu}$, Rothemberg ME. Refining the definition of hypereosinophilic syndrome. J Allergy Clin Immunol 2010;126:45. 
10. Lombardi C, Passalacqua G. Eosinophilia and diseases: clinical revision of 1862 cases. Arch Intern Med 2003; 163:1371.

11. Lee WJ, Lim HK, Lim JH, et al. Foci of eosinophil-related necrosis in the liver: imaging findings and correlation with eosinophilia. AJR Am J Roentgenol 1999;172:1255-61.
12. Yoo SY, Han JK, Kim YH, et al. Focal eosinophilic infiltration in the liver: radiologic findings and clinical course. Abdom Imaging 2003;28:326-32.

13. Jang HJ, Lee WJ, Lee SJ, et al. Focal eosinophilic necrosis of the liver in patients with underlying gastric or colorectal cancer: CT differentiation from metastasis. Korean J Radiol 2002;3:240-4. 